\title{
Thermal Critical Points and Quantum Critical End Point in the Frustrated Bilayer Heisenberg Antiferromagnet
}

\author{
J. Stapmanns, ${ }^{1}$ P. Corboz,${ }^{2}$ F. Mila, ${ }^{3}$ A. Honecker, ${ }^{4}$ B. Normand, ${ }^{5}$ and S. Wessel ${ }^{1}$ \\ ${ }^{1}$ Institut für Theoretische Festkörperphysik, JARA-FIT and JARA-HPC, RWTH Aachen University, 52056 Aachen, Germany \\ ${ }^{2}$ Institute for Theoretical Physics and Delta Institute for Theoretical Physics, \\ University of Amsterdam, Science Park 904, 1098 XH Amsterdam, Netherlands \\ ${ }^{3}$ Institute of Physics, Ecole Polytechnique Fédérale Lausanne (EPFL), 1015 Lausanne, Switzerland \\ ${ }^{4}$ Laboratoire de Physique Théorique et Modélisation, CNRS UMR 8089, \\ Université de Cergy-Pontoise, 95302 Cergy-Pontoise Cedex, France \\ ${ }^{5}$ Neutrons and Muons Research Division, Paul Scherrer Institute, 5232 Villigen-PSI, Switzerland
}

(Received 14 June 2018; published 17 September 2018)

\begin{abstract}
We consider the finite-temperature phase diagram of the $S=1 / 2$ frustrated Heisenberg bilayer. Although this two-dimensional system may show magnetic order only at zero temperature, we demonstrate the presence of a line of finite-temperature critical points related to the line of first-order transitions between the dimer-singlet and -triplet regimes. We show by high-precision quantum Monte Carlo simulations, which are sign-free in the fully frustrated limit, that this critical point is in the Ising universality class. At zero temperature, the continuous transition between the ordered bilayer and the dimer-singlet state terminates on the first-order line, giving a quantum critical end point, and we use tensor-network calculations to follow the first-order discontinuities in its vicinity.
\end{abstract}

DOI: 10.1103/PhysRevLett.121.127201

The concept of the critical point is ubiquitous in statistical thermodynamics. One may need look no further than the liquid-gas transition [1] in systems as familiar as water to find examples where a line of first-order transitions terminates as a function of temperature and a control parameter, such as the pressure. Because the phase transitions are discontinuous, the line has no critical properties, but its termination point does. In contrast with this critical point, the term "critical end point" (CEP) is reserved for the situation where a line of continuous transitions terminates on a line of discontinuous ones [2-4]. In this case, critical behavior is present everywhere on the critical line, and it has been proposed that this behavior is reflected in certain properties of the discontinuous boundary on which the line terminates [4].

Quantum spin systems have proven to offer an excellent forum for the experimental and theoretical investigation of phase transitions and critical phenomena. Quantum phase transitions (QPTs) [5], predominantly in low-dimensional systems, have been controlled by pressure [6], applied magnetic field [7,8], and sample disorder [9], and the associated quantum critical regime [5] explored at finite temperatures [10]. Frustrated quantum magnets extend the nature of the available QPTs to include exact ground states $[11,12]$, exotic bound states [13,14], spin liquids [15], and nontrivial topology [16]. Here we consider the frustrated bilayer $S=1 / 2$ antiferromagnet, a two-dimensional (2D) model with Heisenberg exchange.
In this Letter we demonstrate that, although this system has long-ranged magnetic order and spontaneous breaking of SU(2) symmetry only at zero temperature, a line of critical points appears at finite temperature, $T$. As $T$ is increased, each critical point can be understood as the termination of a line of finite- $T$ first-order transitions, exactly like the critical point of the liquid-gas transition, and all have Ising nature. The critical line is associated with a line of first-order transitions at $T=0$, where we show that the phase diagram as a function of frustration contains a quantum critical end point (QCEP), at which a line of continuous transitions terminates on the line formed by the first-order quantum phase transitions.

We are motivated by our study of the frustrated spin ladder [17], and in particular of its perfectly frustrated limit $[13,14]$. Like its 1D analog, the frustrated bilayer has a firstorder transition between dimer-singlet and -triplet regimes, and in the fully frustrated case it has completely flat excitation bands composed of many-particle bound states. However, in 2D, magnetic order is possible at $T=0$, on top of which thermal fluctuations may cause qualitatively different physics to set in.

The model we investigate is represented schematically in the insets of Fig. 1. In addition to the interaction, $J_{\perp}$, defining the dimer unit and the intralayer interaction, $J_{\|}$, defining the two planes of the system, we include a symmetrical, diagonal, and frustrating interlayer coupling, $J_{\times}$. Only antiferromagnetic couplings are considered. The Hamiltonian for any quantum spin $S$ is 


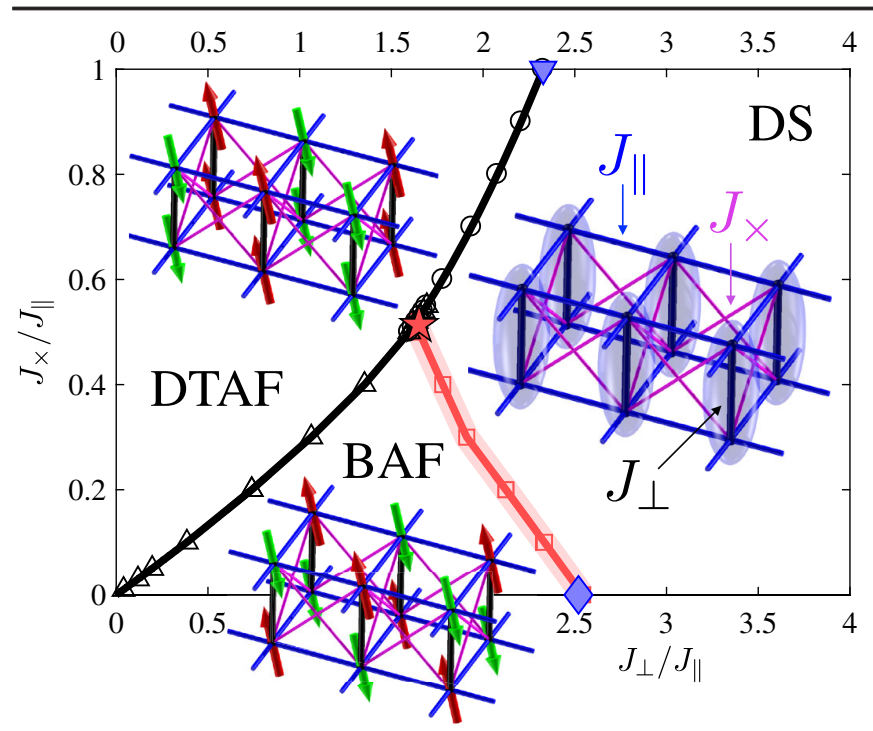

FIG. 1. Phase diagram of the frustrated Heisenberg bilayer at zero temperature. DS: dimer-singlet regime; DTAF: dimer-triplet antiferromagnet; BAF: bilayer antiferromagnet. Insets provide schematic representations of the three phases, where each site hosts an $S=1 / 2$ quantum spin, ellipsoids represent singlet states of two spins, and the Heisenberg couplings between spins are specified by the parameters $J_{\perp}, J_{\|}$, and $J_{\times}$. The line of first-order transitions from DTAF to DS or BAF phases is shown in black and the line of second-order transitions from the DS phase to the BAF phase in red; red shading indicates the error bars in our calculations. Blue symbols denote the QPTs in the unfrustrated (UFB, diamond) and fully frustrated (FFB, triangle) bilayers. The red star denotes the QCEP, where the red line terminates on the black one.

$$
H=\sum_{i} J_{\perp} \vec{S}_{i, 1} \cdot \vec{S}_{i, 2}+\sum_{\substack{i, m=1,2 \\ j=i+\hat{x}, i+\hat{y}}}\left[J_{\|} \vec{S}_{i, m} \cdot \vec{S}_{j, m}+J_{\times} \vec{S}_{i, m} \cdot \vec{S}_{j, \bar{m}}\right]
$$

where $i$ is the dimer bond index, $j$ denotes the nearestneighbor dimers in the bilayer, $m=1$ and 2 denote the two layers, and $\bar{m}$ is the layer opposite to $m$.

Our initial focus is the fully frustrated bilayer (FFB), $J_{\times}=J_{\|}$. In this situation, Eq. (1) can be reexpressed as

$$
H=J_{\|} \sum_{i, j} \vec{T}_{i} \cdot \vec{T}_{j}+J_{\perp} \sum_{i}\left[\frac{1}{2} \vec{T}_{i}^{2}-S(S+1)\right]
$$

where $\vec{T}_{i}=\vec{S}_{i, 1}+\vec{S}_{i, 2}$ is the total spin of dimer $i[18,19]$. Clearly Eq. (2) has one purely local conservation law, on $\vec{T}_{i}^{2}$, for every dimer in the system. Henceforth we restrict our considerations to the case $S=1 / 2$. Thus $T_{i}$ takes the values 0 [a dimer singlet (DS), indicated by the ellipsoids in Fig. 1] or 1 [a dimer triplet (DT)]. For a given set $\left\{T_{i}\right\}$, the first term of Eq. (2) is the Hamiltonian of an open $n$-site spin-1 cluster, which is nonzero only for groups of $n \geq 2$ neighboring DTs; the second term counts these DTs relative to DSs.

As first noted [20] for the fully frustrated $S=1 / 2$ ladder, the model of Eq. (2) possesses a first-order DS-to-DT QPT as a function of the coupling ratio $J_{\perp} / J_{\|}$; the two possible ground states are characterized by all $T_{i}=0$, when $J_{\perp}$ is dominant, or all $T_{i}=1$ when the combination of $J_{\|}$and $J_{\times}$ forces the creation of DTs. For the FFB, the ground state in the DT phase exhibits long-range antiferromagnetic order of the triplet states, which we denote DTAF. Based on energy arguments comparing the DS state with the spin-1 square-lattice Heisenberg model equivalent to the DTAF state, this transition is known to occur at $J_{\perp, c}=2.3279(1) J_{\|}[21]$. Several authors have studied this system, notably by the construction of exact states [22,23] and in a magnetic field [24-26], and its geometry is realized in the material $\mathrm{Ba}_{2} \mathrm{CoSi}_{2} \mathrm{O}_{6} \mathrm{Cl}_{2}$ [27,28], albeit with predominantly $X Y$ interactions.

We use stochastic series expansion [29] quantum Monte Carlo (QMC) simulations with directed loop updates $[30,31]$ to examine the thermodynamic properties of the FFB in the vicinity of the QPT. It has been shown recently $[13,17,32,33]$ that QMC methods can be applied to such highly frustrated systems by expressing the Hamiltonian in the dimer basis [Eq. (2)]. The sign problem is entirely absent in perfectly frustrated models, including the FFB, and is only moderately serious over a wide range of coupling ratios corresponding to imperfect frustration, as we show in Sec. S1 of the Supplemental Material [34]. Combined with a parallel tempering approach [13], required to enhance state mixing in the vicinity of the first-order QPT, we access system sizes $2 \times L \times L$ up to $L=48$ within the temperature regime relevant for the critical point $\left(T \gtrsim 0.3 J_{\|}\right)$. At lower temperatures, strong hysteresis effects appear for couplings close to the QPT.

The thermodynamic properties obtained from QMC simulations for the FFB are shown in Fig. 2. The magnetic susceptibility, $\chi(T)$ [Fig. 2(a)], provides a clear characterization of the gapped DS phase for $J_{\perp}>J_{\perp, c}$, namely, an exponentially rapid rise to a broad peak, and of the DTAF phase for $J_{\perp}<J_{\perp, c}$, where $\chi$ approaches a finite value at low $T$; this constant is the same as for the spin-1 Heisenberg model on the square lattice. The first hint of critical-point behavior is provided by the energy [Fig. 2(b)], which shows a clear discontinuity as a function of the coupling ratio at lower temperatures, but a continuous evolution at higher ones. To examine this in more detail we consider the dimer singlet density, $\rho_{s}=\left\langle N_{s}\right\rangle / N_{d}$, where $N_{d}$ is the number of dimer $\left(J_{\perp}\right)$ bonds and $N_{s}=\sum_{i} P_{s, i}$ the number operator for singlets on these bonds, $P_{s, i}$ being a local singlet projector on bond $i$; the DT density is simply $\rho_{t}=1-\rho_{s}$. In the ground state, $\rho_{s}$ jumps directly from 0 to 1 at $J_{\perp, c}$. We observe [Fig. 2(d)] that this discontinuity persists up to $T \simeq 0.54 J_{\|}$, whereas $\rho_{s}$ varies smoothly across $J_{\perp, c}$ at 

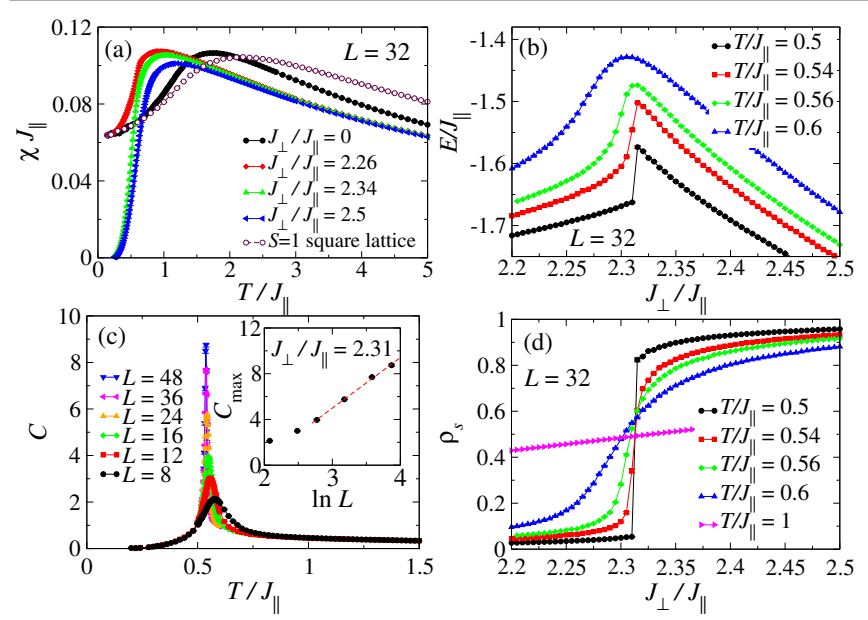

FIG. 2. Thermodynamic properties of the FFB determined from QMC simulations. (a) Magnetic susceptibility, $\chi(T)$, shown for a wide range of coupling ratios. (b) Energy, $E$, as a function of $J_{\perp} / J_{\|}$for different temperatures. (c) Specific heat, $C(T)$, computed at $J_{\perp} / J_{\|}=2.31$. Inset: Finite-size scaling of peak height, $C_{\max }$. (d) Singlet density, $\rho_{s}$, as a function of $J_{\perp} / J_{\|}$for different temperatures.

higher $T$. Thus although magnetic order is found only in the DTAF at $T=0$, the transition from predominantly DT to predominantly DS character persists as a first-order line to finite temperatures, of the same order as the interaction parameters, before terminating at an apparent critical point.

To rationalize the appearance of critical-point physics, we note that the singlet and triplet states on each dimer unit form a binary degree of freedom. This effective Ising variable corresponds to the two distinct irreducible representations of the spin in the two-site unit cell $(2 \otimes 2=1 \oplus 3)$. The line of first-order transitions from DS- to DT-dominated states at finite temperatures may thus terminate at a finite- $T$ Ising critical point, which resembles the liquid-gas transition. This result reflects a key additional property of the $\mathrm{SU}(2)$-symmetric frustrated bilayer model. Although the continuous symmetry precludes a finite order parameter at $T>0$, thermal fluctuations of the binary variable, whose origin lies in the two-site nature of the unit cell, nevertheless stabilize a critical point.

To identify this Ising critical point in the FFB, we employ finite-size scaling of several thermodynamic quantities. In Fig. 2(c) we show that the specific heat, $C(T)$, computed at $J_{\perp} \approx J_{\perp, c}$, develops a sharp peak at $T \simeq 0.55 J_{\|}$. The logarithmic form [41] of the size-scaling of the peak height $\left(C_{\max }\right.$, shown in the inset) indicates that the transition we observe is consistent with emerging Ising universality.

Our most accurate means of locating the critical point is to compute the singlet susceptibility, $\chi_{s}=\beta / N_{d}\left(\left\langle N_{s}^{2}\right\rangle-\right.$ $\left\langle N_{s}\right\rangle^{2}$ ). Figure 3(a) shows that $\chi_{s}(T)$, computed for a value of $J_{\perp} / J_{\|}$very near our final estimate of the critical point and for a number of system sizes, also shows a sharp peak at the same temperature. The inset shows the
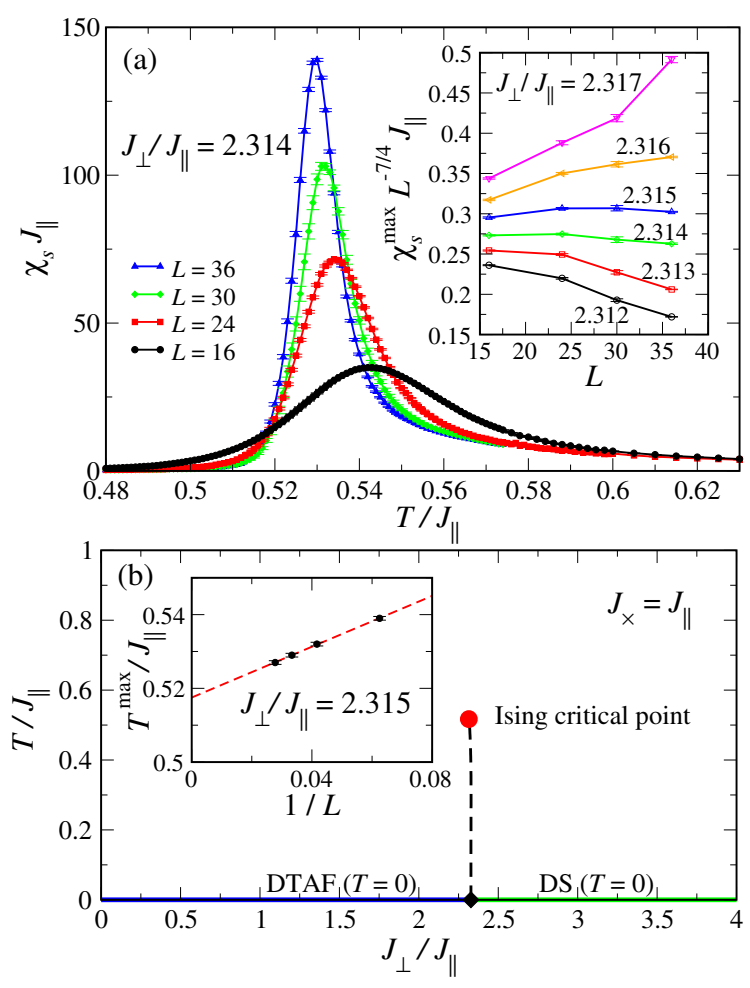

FIG. 3. (a) Singlet susceptibility, $\chi_{s}(T)$, computed for systems of different sizes, $L$. Inset: Finite-size scaling of the rescaled peak height for different values of $J_{\perp} / J_{\|}$. (b) Phase diagram of the FFB. The dashed line marks the finite-temperature first-order transition and the red dot the Ising critical point, $\left(J_{\perp, I}, T_{I}\right)=$ $\left(2.315(1) J_{\|}, 0.517(3) J_{\|}\right)$. Blue and green colors represent, respectively, the pure DTAF and DS phases at $T=0$, where the QPT occurs at $J_{\perp, c}=2.3279(1) J_{\|}$. Inset: Finite-size scaling of the temperature, $T^{\max }$, of the peak in $\chi_{s}(T)$ for coupling ratio $J_{\perp} / J_{\|}=2.315$.

dependence on $L$ of the peak maximum, $\chi_{s}^{\max }$, scaled by $L^{7 / 4}$ [42], where the curve becoming flat (around $\left.J_{\perp} / J_{\|}=2.315\right)$ is in accord with 2D Ising universality. At smaller (larger) values of $J_{\perp} / J_{\|}$, the rescaled $\chi_{s}^{\max }$ bends downwards (upwards) with increasing $L$, indicative of subcritical (first-order) behavior.

We draw the coupling-temperature phase diagram of the FFB in Fig. 3(b). Our estimate of the Ising critical point is $\left(J_{\perp, I}, T_{I}\right)=\left(2.315(1) J_{\|}, 0.517(3) J_{\|}\right)$, where $T_{I}$ is based on finite-size scaling of the form $T^{\max }(L)-T_{I} \propto 1 / L^{\nu}$, with $\nu=1$ for 2D Ising criticality [43] [inset, Fig. 3(b)]. Although this first-order line appears to be very steep on the scale of Fig. 3(b) $\left(J_{\perp, c}=2.3279(1) J_{\|}\right.$at $T=0$ [21]), its precise shape is a nontrivial consequence of the interplay between quantum and thermal fluctuations, which we analyze in Sec. S2 of the Supplemental Material [34].

To address the generality of this critical-point phenomenology, we consider the bilayer model away from perfect frustration. We first draw the ground-state phase diagram connecting the FFB to its unfrustrated counterpart (Fig. 1). 

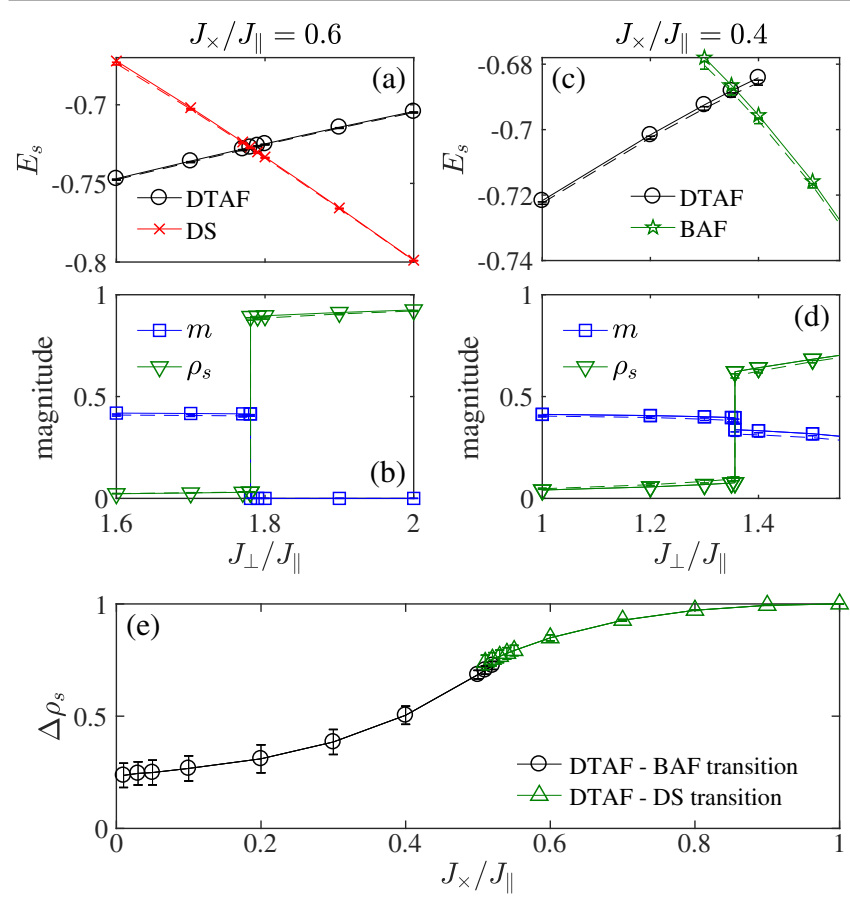

FIG. 4. (a) Energies of the DTAF and DS phases as functions of $J_{\perp} / J_{\|}$at $J_{\times} / J_{\|}=0.6$; the line-crossing marks the first-order transition. Full lines show (simple-update) iPEPS results with $D=10$, dashed lines the $D \rightarrow \infty$ extrapolated results. (b) Corresponding singlet density, $\rho_{s}$, and local magnetic moment, $m$. (c),(d) As for (a) and (b) with $J_{\times} / J_{\|}=0.4$, where the transition is between DTAF and BAF. (e) Discontinuity in $\rho_{s}$, taken from data extrapolated in $D$, shown along the entire first-order transition line of Fig. 1.

The unfrustrated bilayer (UFB) also has two phases at $T=0$, an ordered $S=1 / 2$ bilayer AF (BAF) at small $J_{\perp}$ and a DS phase otherwise. This model has been studied extensively, including in Refs. [44-48], and the QPT is known to occur at $J_{\perp} / J_{\|}=2.5220(2)$ [48]. This transition is second-order, with $3 \mathrm{D} \mathrm{O}(3)$ universality and continuous growth of the BAF order parameter, which is quite different from that of the DTAF (insets, Fig. 1).

We compute the ground-state phase diagram by the method of infinite projected entangled pair states (iPEPS) [49-51], which is a variational tensor-network ansatz for a 2D wave function in the thermodynamic limit. As we discuss in Sec. S3 of the Supplemental Material [34], the accuracy of this technique can be controlled systematically by the bond dimension, $D$, of the tensors, and tensor optimization was performed using both the simple- $[52,53]$ and full-update approaches [51,54]. Estimates of energies, singlet densities, and magnetic order parameters in the limit of infinite $D$ were obtained by extrapolation in $1 / D$ [55], as illustrated in the Supplemental Material [34]. We show our results in Figs. 4(a) and 4(b) for a constant frustration ratio $J_{\times} / J_{\|}=0.6$ and in Figs. 4(c) and 4(d) for $J_{\times} / J_{\|}=0.4$. A discontinuous transition is evident in both cases.
Critical couplings for the first-order transition line were determined from the intersection of the energies of the respective phases [Figs. 4(a) and 4(c)]. The second-order transition line was determined from the vanishing of the BAF order parameter (obtained by full-update optimization and extrapolation). We find that the phase diagram, shown in Fig. 1, possesses a first-order transition, out of the DTAF phase, for all values of $J_{\times} / J_{\|}$. The line of continuous BAF-to-DS transitions extends from the UFB transition to the point $J_{\perp}=1.638(15) J_{\|}, J_{\times}=0.520(5) J_{\|}$, where it terminates on the first-order line. By the definition of Refs. [2,4], this is a QCEP - a CEP occurring at $T=0$. The term QCEP has been applied by some authors to fieldinduced magnetic transitions in heavy-fermion systems, apparently to describe critical-point physics (termination of a first-order line) [56], but not in all discussions of the same topic [57].

To our knowledge, there has been very little discussion of the QCEP. In studies of the CEP [4], it is proposed that the critical properties of the terminating line should be reflected in the properties of the discontinuities on the firstorder line in the vicinity of the CEP. Unfortunately, we are not presently able to perform finite- $T$ calculations in the vicinity of the QCEP [34]. However, from calculations of $\rho_{s}$, of the type shown in Figs. 4(b) and 4(d), we are able to deduce the size of the discontinuity, $\Delta \rho_{s}$, along the firstorder line at $T=0$ [Fig. 4(e)]. Because $\rho_{s}$ is related to the energy density, no jump is expected in $\Delta \rho_{s}$ on passing through the QCEP, due to the continuous nature of the BAF-DS transition. While $\Delta \rho_{s}$ is indeed continuous within our error bars, our data do suggest a discontinuity in its slope across the QCEP. Certainly the critical properties around the QCEP pose a challenge to presently available numerical methods.

The limit of weak $J_{\perp}$ and $J_{\times}$is of special interest in the frustrated ladder, where the DT-to-DS transition may become continuous [58] and there have been proposals [59] of an intermediate phase. In the frustrated bilayer, our calculations show that the first-order nature of the transition is robust, with finite jumps in the singlet density [Fig. 4(e)] all the way to $J_{\perp}=J_{\times}=0$. The value of $\Delta \rho_{s}$ in this limit can be understood from the convergence of $\rho_{s}$ to $1 / 4$ as $J_{\perp} \rightarrow 0$ in the UFB, where the two layers of the BAF become uncorrelated, but the immediate vanishing of $\rho_{s}$ when any finite $J_{\times}$at $J_{\perp}=0$ stabilizes the triplet state. We conclude that the $2 \mathrm{D}$ system remains more conventional in this regard than the $1 \mathrm{D}$ case.

Returning now to the finite- $T$ Ising critical point, we expect this to persist all the way across the phase diagram of Fig. 1 because of its association with the first-order transition. For confirmation, we perform QMC simulations at $J_{\times}=0.7 J_{\|}$, where the sign problem remains moderate. As shown in Sec. S1 of the Supplemental Material [34], our results establish that the critical point is still present, occurring at $T=0.45(1) J_{\|}$. While we are unable by QMC 
simulations to study the first-order DTAF-to-BAF transition line, our iPEPS calculations of $\rho_{s}$ indicate that the binary character of the dimer spin is preserved. We stress that the physics of this line of critical points is a consequence not only of the first-order line but also of the Ising degree of freedom arising due to the dimer-based unit cell.

In summary, we have shown that the frustrated $S=1 / 2$ bilayer with only Heisenberg interactions possesses a line of finite-temperature critical points related to a line of firstorder transitions in its zero-temperature phase diagram. A second line, of continuous transitions from the rung-singlet to the bilayer-ordered phase, terminates on the first line, creating a QCEP. Understanding the critical properties around the QCEP sets a challenge for theory and numerics both in $2 \mathrm{D}$ and in higher dimensions.

This work was supported by the Deutsche Forschungsgemeinschaft (DFG) in the framework of Grants FOR 1807 and RTG 1995, the Swiss National Science Foundation (SNF), and the European Research Council (ERC) under the EU Horizon 2020 research and innovation program (Grant No. 677061). We thank the IT Center at RWTH Aachen University and the JSC Jülich for access to computing time through JARA-HPC.

[1] C. C. de la Tour, Ann. Chim. Phys. 21, 127 (1822).

[2] P. M. Chaikin and T. C. Lubensky, Principles of Condensed Matter Physics (Cambridge University Press, Cambridge, England, 1995), p. 665.

[3] M. E. Fisher and P. J. Upton, Phys. Rev. Lett. 65, 2402 (1990).

[4] M. E. Fisher and M. C. Barbosa, Phys. Rev. B 43, 11177 (1991).

[5] S. Sachdev, Quantum Phase Transitions (Cambridge University Press, Cambridge, England, 2011).

[6] C. Rüegg, B. Normand, M. Matsumoto, A. Furrer, D. F. McMorrow, K. W. Krämer, H.-U. Güdel, S. N. Gvasaliya, H. Mutka, and M. Boehm, Phys. Rev. Lett. 100, 205701 (2008).

[7] T. Giamarchi, C. Rüegg, and O. Tchernyshyov, Nat. Phys. 4, 198 (2008), and references therein.

[8] B. Thielemann, C. Rüegg, H. M. Rønnow, A. M. Läuchli, J.-S. Caux, B. Normand, D. Biner, K. W. Krämer, H.-U. Güdel, J. Stahn, K. Habicht, K. Kiefer, M. Boehm, D. F. McMorrow, and J. Mesot, Phys. Rev. Lett. 102, 107204 (2009).

[9] R. Yu, L. Yin, N. S. Sullivan, J. S. Xia, C. Huan, A. PaduanFilho, N. F. Oliveira Jr., S. Haas, A. Steppke, C. F. Miclea, F. Weickert, R. Movshovich, E.-D. Mun, B. L. Scott, V. S. Zapf, and T. Roscilde, Nature (London) 489, 379 (2012).

[10] P. Merchant, B. Normand, K. W. Krämer, M. Boehm, D. F. McMorrow, and C. Rüegg, Nat. Phys. 10, 373 (2014).

[11] C. K. Majumdar and D. Ghosh, J. Math. Phys. 10, 1388 (1969).

[12] B.S. Shastry and B. Sutherland, Physica (Amsterdam) 108B+C, 1069 (1981).
[13] A. Honecker, S. Wessel, R. Kerkdyk, T. Pruschke, F. Mila, and B. Normand, Phys. Rev. B 93, 054408 (2016).

[14] A. Honecker, F. Mila, and B. Normand, Phys. Rev. B 94, 094402 (2016).

[15] L. Savary and L. Balents, Rep. Prog. Phys. 80, 016502 (2017).

[16] J. Romhányi, K. Penc, and R. Ganesh, Nat. Commun. 6, 6805 (2015).

[17] S. Wessel, B. Normand, F. Mila, and A. Honecker, SciPost Phys. 3, 005 (2017).

[18] Y. Xian, Phys. Rev. B 52, 12485 (1995).

[19] A. Honecker, F. Mila, and M. Troyer, Eur. Phys. J. B 15, 227 (2000).

[20] M. P. Gelfand, Phys. Rev. B 43, 8644 (1991).

[21] E. Müller-Hartmann, R. R. P. Singh, C. Knetter, and G. S. Uhrig, Phys. Rev. Lett. 84, 1808 (2000).

[22] H. Q. Lin and J. L. Shen, J. Phys. Soc. Jpn. 69, 878 (2000).

[23] H. Q. Lin, J. L. Shen, and H. Y. Shik, Phys. Rev. B 66, 184402 (2002).

[24] J. Richter, O. Derzhko, and T. Krokhmalskii, Phys. Rev. B 74, 144430 (2006).

[25] O. Derzhko, J. Richter, A. Honecker, and H.-J. Schmidt, Low Temp. Phys. 33, 745 (2007).

[26] O. Derzhko, T. Krokhmalskii, and J. Richter, Phys. Rev. B 82, 214412 (2010).

[27] H. Tanaka, N. Kurita, M. Okada, E. Kunihiro, Y. Shirata, K. Fujii, H. Uekusa, A. Matsuo, K. Kindo, and H. Nojiri, J. Phys. Soc. Jpn. 83, 103701 (2014).

[28] J. Richter, O. Krupnitska, V. Baliha, T. Krokhmalskii, and O. Derzhko, Phys. Rev. B 97, 024405 (2018).

[29] A. W. Sandvik, Phys. Rev. B 59, R14157 (1999).

[30] O. F. Syljuåsen and A. W. Sandvik, Phys. Rev. E 66, 046701 (2002).

[31] F. Alet, S. Wessel, and M. Troyer, Phys. Rev. E 71, 036706 (2005).

[32] F. Alet, K. Damle, and S. Pujari, Phys. Rev. Lett. 117, 197203 (2016).

[33] K.-K. Ng and M.-F. Yang, Phys. Rev. B 95, 064431 (2017).

[34] See Supplemental Material at http://link.aps.org/ supplemental/10.1103/PhysRevLett.121.127201, which contains Refs. [35-40], for details on the QMC sign problem, the finite temperature transition line of the FFB model, and the iPEPS calculations.

[35] D. C. Johnston, R. J. McQueeney, B. Lake, A. Honecker, M. E. Zhitomirsky, R. Nath, Y. Furukawa, V. P. Antropov, and Y. Singh, Phys. Rev. B 84, 094445 (2011).

[36] P. Corboz, T. M. Rice, and M. Troyer, Phys. Rev. Lett. 113, 046402 (2014).

[37] T. Nishino and K. Okunishi, J. Phys. Soc. Jpn. 65, 891 (1996).

[38] R. Orús and G. Vidal, Phys. Rev. B 80, 094403 (2009).

[39] S. Singh, R. N. C. Pfeifer, and G. Vidal, Phys. Rev. B 83, 115125 (2011).

[40] B. Bauer, P. Corboz, R. Orús, and M. Troyer, Phys. Rev. B 83, 125106 (2011).

[41] L. Onsager, Phys. Rev. 65, 117 (1944).

[42] D. P. Landau, Phys. Rev. B 13, 2997 (1976).

[43] M. E. Fisher and R. J. Burford, Phys. Rev. 156, 583 (1967).

[44] K. Hida, J. Phys. Soc. Jpn. 61, 1013 (1992).

[45] A. J. Millis and H. Monien, Phys. Rev. Lett. 70, 2810 (1993).

[46] A. W. Sandvik and D. J. Scalapino, Phys. Rev. Lett. 72, 2777 (1994). 
[47] T. Sommer, M. Vojta, and K. W. Becker, Eur. Phys. J. B 23, 329 (2001).

[48] L. Wang, K. S. D. Beach, and A. W. Sandvik, Phys. Rev. B 73, 014431 (2006).

[49] F. Verstraete and J. I. Cirac, arXiv:cond-mat/0407066.

[50] Y. Nishio, N. Maeshima, A. Gendiar, and T. Nishino, arXiv: cond-mat/0401115.

[51] J. Jordan, R. Orús, G. Vidal, F. Verstraete, and J. I. Cirac, Phys. Rev. Lett. 101, 250602 (2008).

[52] G. Vidal, Phys. Rev. Lett. 91, 147902 (2003).

[53] H. C. Jiang, Z. Y. Weng, and T. Xiang, Phys. Rev. Lett. 101, 090603 (2008).
[54] H. N. Phien, J. A. Bengua, H. D. Tuan, P. Corboz, and R. Orús, Phys. Rev. B 92, 035142 (2015).

[55] P. Corboz and F. Mila, Phys. Rev. B 87, 115144 (2013).

[56] S. A. Grigera, R. S. Perry, A. J. Schofield, M. Chiao, S. R. Julian, G. G. Lonzarich, S. I. Ikeda, Y. Maeno, A. J. Millis, and A. P. Mackenzie, Science 294, 329 (2001).

[57] D. Belitz, T. R. Kirkpatrick, and J. Rollbühler, Phys. Rev. Lett. 94, 247205 (2005).

[58] T. Hikihara and O. A. Starykh, Phys. Rev. B 81, 064432 (2010).

[59] O. A. Starykh and L. Balents, Phys. Rev. Lett. 93, 127202 (2004). 


\title{
Supplemental Material for "Thermal Critical Points and Quantum Critical End Point in the Frustrated Bilayer Heisenberg Antiferromagnet"
}

\author{
J. Stapmanns, P. Corboz, F. Mila, A. Honecker, B. Normand, and S. Wessel
}

\section{S1. QMC sign problem}

The accuracy of our thermodynamic calculations (Figs. 2 and 3 of the main text) is attainable because the FFB is amenable to sign-problem-free QMC simulations. These are based upon the very simple form of the Hamiltonian at $J_{\times}=J_{\|}$[Eq. (2) of the main text] when expressed in the dimer spin basis,

$$
\vec{T}_{i}=\vec{S}_{i, 1}+\vec{S}_{i, 2}
$$

of the $J_{\perp}$ bonds. On moving away from this fully frustrated limit, the Hamiltonian takes the form [S1, S2]

$$
\begin{gathered}
H=\frac{1}{2}\left(J_{\|}+J_{\times}\right) \sum_{i, j} \vec{T}_{i} \cdot \vec{T}_{j}+\frac{1}{2}\left(J_{\|}-J_{\times}\right) \sum_{i, j} \vec{D}_{i} \cdot \vec{D}_{j} \\
+J_{\perp} \sum_{i}\left[\frac{1}{2} \vec{T}_{i}^{2}-S(S+1)\right],
\end{gathered}
$$

which for $J_{\times} \neq J_{\|}$contains the spin-difference operators

$$
\vec{D}_{i}=\vec{S}_{i, 1}-\vec{S}_{i, 2}
$$

The $D D$ terms cause the sign problem to reappear.

As discussed for the frustrated ladder in Ref. [S1], a 1D system with only $D D$ (and no $D T$ ) terms has only a mild sign problem over much of the $J_{\times} \neq J_{\|}$phase diagram. This can be traced to the fact that QMC configurations with a negative sign are rare with respect to the full configuration space and are completely absent for a ladder with open boundary conditions [S2]. For a finite ladder with periodic boundary conditions, negative-weight configurations contain a string of bond operators, when formulated within the stochastic series expansion, that spans the full spatial extent of the system, and the probability for such configurations is suppressed strongly by increasing the system size at low temperatures.

By contrast, negative-weight QMC configurations occurring in the frustrated bilayer may contain bond operators from only a small part of the lattice, i.e., in $2 \mathrm{D}$ these configurations need not span the system and do appear with open boundary conditions. Hence we do suffer from strong sign problems in our QMC simulations sufficiently far from the fully frustrated limit. To examine the onset of the sign problem in more detail, we have performed a scan, over the full parameter space of the Hamiltonian in Eq. (S2), to measure the average sign, $\langle$ sign $\rangle$, of the QMC configurations sampled. The results obtained for a moderate system size, $L=10$, and at a temperature of $T=0.05 J_{\|}$, are represented by the color scale in Fig. S1. The behavior of $\langle$ sign $\rangle$ is correlated with

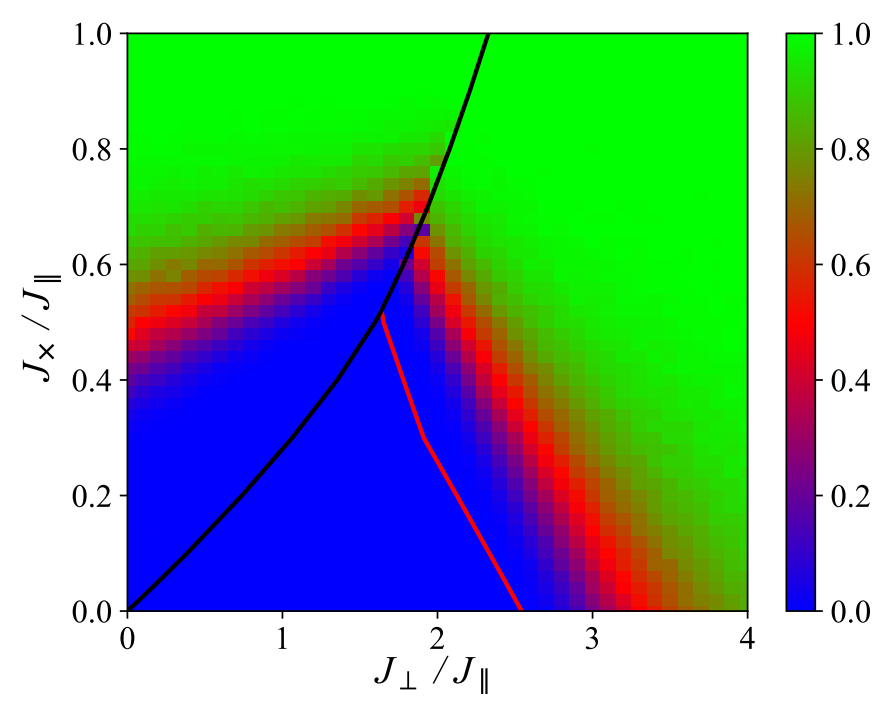

FIG. S1. Calculation of $\langle\operatorname{sign}\rangle$, with $L=10$ and $T=0.05 J_{\|}$, within a dimer-basis formulation of the QMC algorithm. Solid lines reproduce the phase boundaries shown in Fig. 1 of the main text.

the location of the phase boundaries in the ground state (reproduced from Fig. 1 of the main text). Although neither the BAF phase nor the transitions into it are accessible in the dimer basis, the sign problem does remain moderate in large parts of the other two phases.

For practical purposes, a value $\langle$ sign $\rangle \gtrsim 0.1$ can still be tolerated in our QMC simulations, i.e., compensated by increasing the QMC sampling (the CPU time) by a factor of 100. As an example of working at partial frustration, we illustrate our estimation of the critical temperature at frustration ratio $J_{\times}=0.7 J_{\|}$(reported in the main text). As in Fig. 2(d) of the main text, we computed the singlet density, $\rho_{s}$, at different temperatures as a function of the coupling ratio $J_{\perp} / J_{\|}$, achieving a maximum system size of $L=24$. As Fig. S2 makes clear, we observe a clear jump in $\rho_{s}$ near $J_{\perp} / J_{\|}=1.93$ for temperatures $T \leq 0.44 J_{\|}$, whereas this quantity exhibits a continuous growth for $T \geq 0.46 J_{\|}$. Thus we estimate the location of the critical point at $J_{\times}=0.7 J_{\|}$ to be $\left(J_{\perp, I}, T_{I}\right)=\left(1.93(1) J_{\|}, 0.45(1) J_{\|}\right)$. We comment that the corresponding first-order transition point in the ground state, which we located by our iPEPS calculations, is $J_{\perp, c}=1.9375(8) J_{\|}$, and hence the finite- $T$ first-order line at $J_{\times}=0.7 J_{\|}$shows the same weak $T$ dependence as at full frustration.

With current computer power it becomes practically impossible to perform reliable QMC simulations beyond 


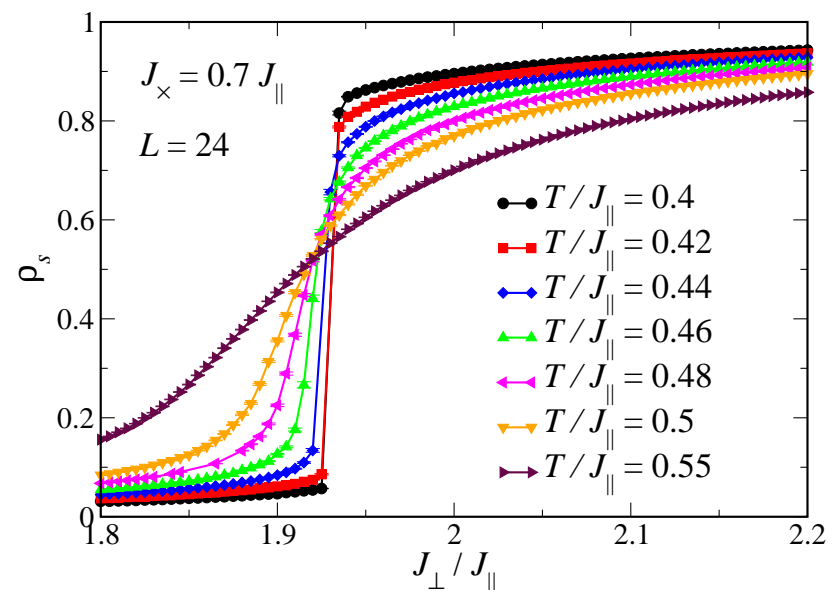

FIG. S2. Singlet density, $\rho_{s}$, calculated on a system of size $L=24$ with frustration ratio $J_{\times}=0.7 J_{\|}$, shown for different temperatures as a function of coupling ratio $J_{\perp} / J_{\|}$.

the regime represented in red in Fig. S1. In the present case, the sign problem prevents us from studying the region of the QCEP by QMC, and also from following the line of finite-temperature Ising critical points below frustration values $J_{\times} / J_{\|} \approx 0.7$. For either purpose, accurate thermodynamic studies would require system sizes considerably larger than that used in Fig. S1. For future analysis it may be necessary to investigate whether the sign problem can be alleviated in the vicinity of the QCEP by further optimizing the local computational basis using a more general unitary transformation. We comment in closing that the UFB at $J_{\times}=0$ is a bipartite system, which can be studied in detail by QMC simulations in the conventional site basis without encountering a sign problem, and indeed many previous studies of the UFB have been performed in this way, including in Refs. [S3, S4].

\section{S2. Finite-temperature transition line}

Here we discuss in detail the form, in the space of coupling ratio and temperature, of the finite- $T$ first-order line in the FFB. As noted in the main text, in general one expects the level crossing between the DT- and DSdominated phases to move to a different coupling ratio as a function of temperature, which influences the two phases quite differently. However, it is clear in Fig. 3(b) of the main text that the effects of thermal fluctuations are not very strong on the scale of the entire parameter space.

For a full analysis of the problem, we consider the free energies on the two sides of the transition line, $F_{1}(x, T)$ and $F_{2}(x, T)$, where $x=J_{\perp} / J_{\|}$denotes the coupling ratio in the FFB. Let 1 denote the DTAF phase, which is gapless, and 2 the DS phase, which is gapped. At finite temperature, $F_{i}(x, T)=E_{i}(x, T)-T S_{i}(T)(i=1,2)$, where $E_{1}(x, 0)$ and $E_{2}(x, 0)$ are the ground-state energies

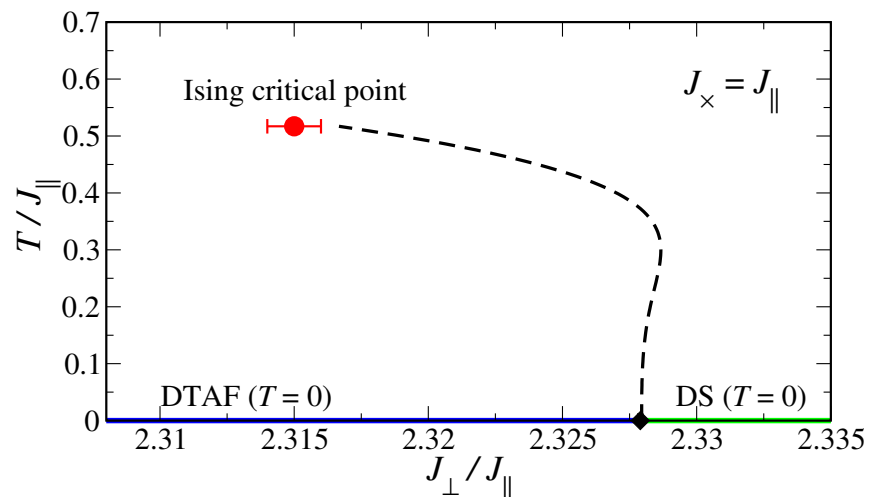

FIG. S3. Phase diagram of the FFB showing in detail the region of the finite- $T$ first-order transition line (dashed). As in Fig. 3(b) of the main text, the red dot marks the Ising critical point, $\left(J_{\perp, I}, T_{I}\right)=\left(2.315(1) J_{\|}, 0.517(3) J_{\|}\right)$, computed by QMC and the black dot the QPT at $\left(J_{\perp, c}, T\right)=$ $\left(2.3279(1) J_{\|}, 0\right)$, taken from Ref. [S5].

and we take $S_{i}(T)$ to be $x$-independent in the vicinity of the QPT. Because the model is in two spatial dimensions, the specific heat of the DTAF should obey $C_{v}(T)=a T^{2}$, whence by integration

$$
E_{1}(x, T)-E_{1}(x, 0)=\frac{1}{3} a T^{3} \text { and } S_{1}(T)=\frac{1}{2} a T^{2},
$$

giving to a good approximation

$$
F_{1}(x, T)=E_{1}(x, 0)-\frac{1}{6} a T^{3} .
$$

In contrast to these power-law expressions, in the gapped DS phase one expects only exponentially weak changes in $E_{2}(x, T)$ and $S_{2}(T)$. By approximating the well-gapped DS state using the response of a single dimer with the same spin gap, $\Delta$, we obtain

$$
F_{2}(x, T)=-T \ln Z_{2}(x, T)
$$

with

$Z_{2}(x, T)=\exp \left[-E_{2}(x, 0) / T\right]+3 \exp \left[-\left(E_{2}(x, 0)+\Delta\right) / T\right]$,

leading to

$$
F_{2}(x, T)=E_{2}(x, 0)-T \ln [1+3 \exp (-\Delta / T)] .
$$

By equating the two approximate free energies we obtain the location of the first-order transition line from the equation

$$
\frac{1}{6} a T^{3}-T \ln [1+3 \exp (-\Delta / T)]=E_{1}(x, 0)-E_{2}(x, 0),
$$

where $a$ is the coefficient of the specific heat per spin1 entity in the DTAF, $\Delta=J_{\perp}$ is the gap in the DS phase, and $E_{1}(x, 0)$ and $E_{2}(x, 0)$ are respectively the zero-temperature energies per dimer of the ordered and gapped phases. 
We use the fact that $E_{1}(x, 0)-E_{2}(x, 0)$ is precisely the ground-state energy of the $S=1$ square-lattice antiferromagnet (see Eq. (2) of the main text), and take both this value and the coefficient $a=0.2441$ from the QMC simulations of Ref. [S6]. As Fig. S3 makes clear, these considerations give an excellent account of the position of the finite- $T$ first-order line in the coupling-temperature phase diagram of the FFB. The fundamental features of the line are its vertical approach to $J_{\perp, c}$ as $T \rightarrow 0$ and the location of the critical point to the left of the zerotemperature QPT. However, the latter is actually the result of a competition between the power-law terms in $F_{1}(x, T)$ and the exponential behavior in $F_{2}(x, T)$, which causes the transition line to rise first to the right (thermal fluctuations favor DTs) before bending back to the left (temperatures higher than $T / J_{\|} \approx 0.3$ favor the DS phase). The crossover temperature appears to depend smoothly on $a$ but to vary linearly with $\Delta$, with a coefficient of approximately 0.15 .

We stress once again the very narrow regime of coupling ratios over which these effects take place in the FFB. Nevertheless, the model provides an excellent example of a system in which the interplay of quantum and thermal fluctuations can be studied in the context of a key physical consequence, namely the location of the line of finite- $T$ transitions.

\section{S3. iPEPS}

Infinite projected entangled pair states (iPEPS) are a variational tensor-network ansatz which provides an efficient representation of the ground states of $2 \mathrm{D}$ systems in the thermodynamic limit [S7-S9]. iPEPS may be considered as a generalization of (infinite) matrixproduct states to two dimensions. The ansatz consists of a unit cell of tensors repeated periodically on the lattice. Here we use one tensor per dimer in the bilayer model. Each tensor has one physical index representing the local Hilbert space of the dimer and four auxiliary indices with bond dimension $D$, which connect to the four nearest-neighbor tensors. By using different unitcell sizes, iPEPS can represent states of differing broken translational symmetry. We find that a unit cell with 2 tensors arranged in a checkerboard pattern is sufficient to capture all the phases of the frustrated bilayer model.

For the optimization of the iPEPS wave function (i.e., finding the optimal variational parameters) we use both the simple-update approach [S10], in which the truncation of a bond index is based on a local approximation of the state, and by the more accurate, but computationally more expensive, full-update method [S8, S11], where the entire state is taken into account for the truncation. Contraction of the tensor network, required in the full-update method and to compute expectation values, was performed by a variant [S12] of the corner-transfer-matrix renormalization-group method [S13, S14], in which we
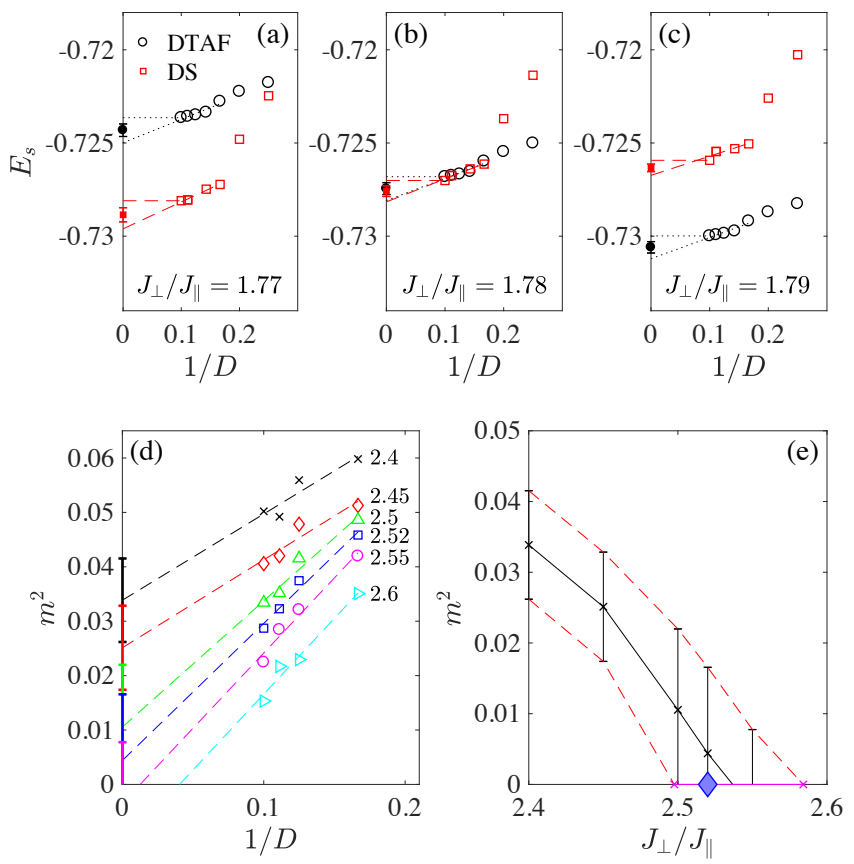

FIG. S4. (a)-(c) Energies of the DTAF and DS states calculated by the simple-update method with $J_{\times} / J_{\|}=0.6$ for three different values of $J_{\perp} / J_{\|}$and shown as functions of the inverse bond dimension. Solid symbols denote the extrapolated estimate in the infinite- $D$ limit. (d) Squared local magnetic moment calculated by the full-update method with $J_{\times}=0$ for the indicated values of $J_{\perp} / J_{\|}$and shown as a function of $1 / D$. (e) Extrapolated value of $m^{2}$ obtained from panel (d). The blue diamond shows the critical coupling obtained by QMC.

exploit the U(1) symmetry of the model [S15, S16]. Further details of iPEPS methods may be found in Refs. [S11, S17].

To illustrate the procedure by which we obtain estimates of physical expectation values from calculations with finite bond dimension, Figs. S4(a)-S4(c) show example data for the energies of the competing DTAF and DS states close to first-order QPT for $J_{\times} / J_{\|}=0.6$ [cf. Fig. 4(a) of the main text]. To estimate the energy in the limit of infinite $D$, we take the average between the value obtained by linear extrapolation in $1 / D$ and the value obtained from our largest $D(=10)$. The former typically provides a lower bound, because the energy converges faster than linearly in $1 / D$, while the latter is an upper bound. As the error bar we take half the difference between the extrapolation and the upper bound (an approach similar to Ref. [S17]). From the intersection of the energy estimates for the two states, taking the error bars into account, we deduce a critical value $J_{\perp} / J_{\|}=1.7803 \pm 0.0014$. For comparison, the value obtained purely from our $D=10$ data is $J_{\perp} / J_{\|}=1.7805$, and thus the finite- $D$ error is very small here.

In Fig. S4(d) we show full-update data for the square of the magnetic order parameter in the $\operatorname{UFB}\left(J_{\times}=0\right)$. 
To obtain an estimate in the infinite- $D$ limit, here we use a linear extrapolation in $1 / D$ and an error bar given by half the difference between the extrapolated and largest$D$ values. Although this procedure provides only a rather crude estimate of the order parameter, we stress that it does allow us to determine the location of the continuous BAF-DS phase transition with reasonable accuracy, as shown in Fig. $\mathrm{S} 4(\mathrm{e})$. In this example we find that the order parameter vanishes at a critical value $J_{\perp} / J_{\|}=2.54(4)$, which is fully compatible with the QMC result, $J_{\perp} / J_{\|}=2.5220(2)[\mathrm{S} 4]$.

[S1] S. Wessel, B. Normand, F. Mila, and A. Honecker, SciPost Phys. 3, 005 (2017).

[S2] F. Alet, K. Damle, and S. Pujari, Phys. Rev. Lett. 117, 197203 (2016).

[S3] A. W. Sandvik and D. J. Scalapino, Phys. Rev. Lett. 72, 2777 (1994).

[S4] L. Wang, K. S. D. Beach, and A. W. Sandvik, Phys. Rev. B 73, 014431 (2006).

[S5] E. Müller-Hartmann, R. R. P. Singh, C. Knetter, and
G. S. Uhrig, Phys. Rev. Lett. 84, 1808 (2000).

[S6] D. C. Johnston, R. J. McQueeney, B. Lake, A. Honecker, M. E. Zhitomirsky, R. Nath, Y. Furukawa, V. P. Antropov, and Y. Singh, Phys. Rev. B 84, 094445 (2011).

[S7] F. Verstraete and J. I. Cirac, arXiv:cond-mat/0407066.

[S8] J. Jordan, R. Orús, G. Vidal, F. Verstraete, and J. I. Cirac, Phys. Rev. Lett. 101, 250602 (2008).

[S9] Y. Nishio, N. Maeshima, A. Gendiar, and T. Nishino, arXiv:cond-mat/0401115.

[S10] H. C. Jiang, Z. Y. Weng, and T. Xiang, Phys. Rev. Lett. 101, 090603 (2008).

[S11] H. N. Phien, J. A. Bengua, H. D. Tuan, P. Corboz, and R. Orús, Phys. Rev. B 92, 035142 (2015).

[S12] P. Corboz, T. M. Rice, and M. Troyer, Phys. Rev. Lett. 113, 046402 (2014).

[S13] T. Nishino and K. Okunishi, J. Phys. Soc. Jpn. 65, 891 (1996).

[S14] R. Orús and G. Vidal, Phys. Rev. B 80, 094403 (2009).

[S15] S. Singh, R. N. C. Pfeifer, and G. Vidal, Phys. Rev. B 83, 115125 (2011).

[S16] B. Bauer, P. Corboz, R. Orús, and M. Troyer, Phys. Rev. B 83, 125106 (2011).

[S17] P. Corboz and F. Mila, Phys. Rev. B 87, 115144 (2013). 